\title{
SÍNDROME DE BURNOUT EM ESTUDANTES DE GRADUAÇÃO DOS CURSOS DE MEDICINA, ENFERMAGEM, ODONTOLOGIA E PSICOLOGIA NO BRASIL: UMA REVISÃO DO PANORAMA BRASILEIRO
}

\author{
BURNOUT SYNDROME AMONG UNDERGRADUATED BRAZILIAN STUDENTS
}

\author{
Leonardo Maso Nassar ${ }^{a^{*}}$, Alisson Maxwell Ferreira Andrade ${ }^{b^{* *}}$, Jorge Luis Sánchez Arévalo ${ }^{c^{* *}}$ \\ leo.mnassar@gmail.com ${ }^{\mathrm{a}}$, alisson.andrade@ufms.br ${ }^{\mathrm{b}}$, jsarevalo@hotmail.com ${ }^{c}$ \\ Escola de Enfermagem de Ribeirão Preto* \\ Universidade Federal do Mato Grosso do Sul ${ }^{* *}$
}

Data do recebimento do artigo: $02 / 05 / 2018$

Data do aceite: $02 / 07 / 2018$

\begin{abstract}
RESUMO
Introdução: A Síndrome de Burnout ou, simplesmente, Burnout é uma síndrome psicológica que surge no cenário de estresse relacionado ao trabalho prolongado, sendo caracterizada como um estado crônico de exaustão emocional, acompanhado por uma tendência para despersonalizar os outros e um senso de realização pessoal diminuído devido ao enfrentamento de situações de trabalho exigentes. As profissões de cuidados de saúde são geralmente consideradas de alto risco para síndrome de Burnout. Do mesmo modo, o Burnout tem sido documentado com altos níveis em estudantes de cursos da área da saúde. Objetivos: mapear a literatura disponível para fornecer uma visão geral sobre os estudos que analisaram Síndrome de Burnout em estudantes de graduação dos cursos da área da saúde em faculdades no Brasil. Materiais e Métodos: Foi realizada uma revisão integrativa da literatura baseada nas diretrizes do Joanna Briggs Institute com a aplicação da mnemônica População, Conceito e Contexto. Resultados: A aplicação dos critérios de elegibilidade possibilitou a seleção final de quinze estudos. Do total, oito estudos analisaram alunos do curso de medicina, cinco estudos analisaram alunos do curso de enfermagem, dois estudos analisaram os alunos do curso de odontologia e nenhum estudo foi encontrado sobre alunos de psicologia. Conclusão: Apesar de ser um assunto de interesse desde 2009, foram observadas diversas carências e oportunidades para estudos futuros, principalmente na área da psicologia.
\end{abstract}

Palavras-chave: Estudantes de medicina; estudantes de enfermagem; estudantes de odontologia; estudante de psicologia; síndrome de burnout; burnout.

\begin{abstract}
Introduction: Burnout Syndrome or simply Burnout is a psychological syndrome that emerges in the stress-related scenario of prolonged work, being characterized as a chronic state of emotional exhaustion, accompanied by a tendency to depersonalize others and a sense of personal fulfillment diminished due to the confrontation of demanding work situations. Health care professions are generally considered to be at high risk for Burnout syndrome. Likewise, Burnout has been documented to high levels in students of health courses. Objectives: To map available literature to provide an overview of the studies that analyzed Burnout Syndrome in undergraduate students of health care courses in colleges in Brazil. Materials and Methods: An integrative literature review based on the Joanna Briggs Institute guidelines was applied with the application of the mnemonic Population, Concept and Context. Results: The application of the eligibility criteria allowed the final selection of fifteen studies. Of the total, eight studies examined medical students, five studies examined nursing course students, two studies examined undergraduate dentistry students and no study was found on psychology students. Conclusion: Despite being a subject of interest since 2009, several shortcomings and opportunities for future studies have been observed, mainly in the area of psychology.
\end{abstract}

Keywords: Students, medical; students, nursing; students, dental; student, psychology; burnout, professional; burnout. 


\section{Introdução}

A formação de um profissional da área da saúde é um desafio para gestores e educadores por conta da complexidade de estimular ao mesmo tempo as habilidades profissionais, interpessoais e humanísticas do aluno ${ }^{1}$. O objetivo das faculdades é proporcionar que estudantes de graduação dos programas de ciências da saúde atinjam as habilidades, conhecimentos e atitudes desejadas para se juntarem à força de trabalho ${ }^{2}$. Portanto, semelhante aos ambientes de trabalho, a graduação requer atividades estruturadas regulares que estão associadas aos níveis esperados de realização do futuro profissional $^{3}$.

Nas profissões de cuidados de saúde, a conclusão do curso de graduação torna-se uma tarefa exigente que pressiona muitos alunos ${ }^{4}$. A própria metodologia de ensino empregada por algumas instituições também pode ser fonte de estresse para o estudante, levando a situações de risco para acadêmicos e pacientes 5 . Caso o aluno possua uma personalidade caracterizada por uma tendência a experimentar emoções e inibições negativas durante as interações sociais ${ }^{6}$, sem o devido apoio emocional, o estudante estará suscetível a desenvolver a Síndrome de Burnout ${ }^{7}$.

A Síndrome de Burnout ou, simplesmente, Burnout é uma síndrome psicológica que surge no cenário de estresse relacionado ao trabalho prolongado, sendo caracterizada como um estado crônico de exaustão emocional, acompanhado por uma tendência para despersonalizar os outros e um senso de realização pessoal diminuído devido ao enfrentamento de situações de trabalho exigentes ${ }^{8}$ ${ }^{9}$. O Burnout apresenta três sintomas fundamentais: exaustão (desgaste, perda de energia, depressão, debilitação e fadiga); sentimentos de cinismo e desprendimento do trabalho (despersonalização, atitudes negativas ou inapropriadas, preocupação isolada, irritabilidade, perda de idealismo e demissão); sensação de ineficácia profissional e falta de realização (reduzida produtividade ou capacidade, baixa moral e incapacidade $)^{10}$. Quando as demandas do ambiente de trabalho são maiores que a capacidade de enfrentamento, ele passa a ser percebido como uma ameaça pelo indivíduo ${ }^{11}$.

As profissões de cuidados de saúde são geralmente consideradas de alto risco para síndrome de Burnout ${ }^{12}$. Tais profissões exigem tempo considerável em intenso envolvimento com outras pessoas, frequentemente com problemas físicos e/ou psicológicos, carregadas de sentimentos como raiva, constrangimento, medo ou frustração ${ }^{13}$. Com efeito, a Síndrome de Burnout é experimentado por atuantes da área da saúde de todos os tipos, incluindo profissionais e estagiários em enfermagem, médicos, dentistas e outros campos de atuação da saúde ${ }^{14}$.

Do mesmo modo, o Burnout tem sido documentado com altos níveis em estudantes de cursos da área da saúde ${ }^{15}$. A gravidade dos problemas de Burnout entre estudantes ilustra os resultados de um estudo de Dyrbye et al. ${ }^{16}$ os autores concluíram que cerca de $50 \%$ dos alunos experimentam Burnout e, entre estudantes de medicina, o Burnout parece estar associado a uma maior probabilidade de ideação suicida. De um modo geral, os estudantes que apresentam os sintomas da Síndrome de Burnout são duas a três vezes mais propensos a terem ideação suicida ${ }^{17}$.

Portanto, reconhecendo os impactos da manifestação da Síndrome de Burnout em estudantes, questiona-se: qual a literatura disponível sobre Síndrome de Burnout em estudantes de graduação dos cursos da área da saúde em faculdades no Brasil? Para solucionar a pergunta da pesquisa, foi realizada uma revisão integrativa da literatura com o objetivo de mapear a literatura disponível para fornecer uma visão geral sobre os estudos que analisaram a Síndrome de Burnout em estudantes de graduação dos cursos da área da saúde em faculdades no Brasil.

\section{Metodologia}

Trata-se de um estudo que utilizou as diretrizes desenvolvidas pela Joanna Briggs Institute $(\mathrm{JBI})^{18}$. O JBI é uma organização internacional sem fins lucrativos que compõe a School of Translational Science of Faculty of Health Science, University of Adelaide na Austrália. Para o presente estudo, foi utilizado a proposta do JBI de Scoping Review que compreende no levantamento das evidências disponíveis para sintetizar o conhecimento sobre um tema.

No estudo em questão, foi aplicada a mnemônica População, Conceito e Contexto (PCC) 
para uma Scoping Review. Dentre os contextos, População refere-se à população ou a um problema que pode ser um indivíduo ou um grupo em uma condição específica; Conceito pode ser todos os elementos detalhados e relevantes a serem considerados; Contexto é determinado segundo o objetivo e a pergunta da revisão, sendo definido pelos fatores culturais. Para a pergunta norteadora do presente estudo, foram definidas:

População: estudantes da área da saúde;

- Conceito: Síndrome de Burnout;

- Contexto: cursos de graduação em faculdades brasileiras.

A escolha pelos cursos a serem analisados foi baseada em Kinser et al. ${ }^{14}$. Portanto, foram analisados os cursos de medicina, enfermagem e odontologia com o acréscimo do curso de psicologia. Com intuito de maximizar a quantidade de estudos encontrados, também foram consideradas como cursos os termos "Ciências da Saúde" e "Saúde Pública".
Quanto à estratégia de busca, foram consultadas seis bases de dados: Scientific Eletronic Library Online (SciELO), Literatura Latino-Americana e do Caribe em Ciências da Saúde (LILACS), PubMed, Cochrane, Scopus e Web of Science. Para adequação das buscas, foram utilizados os descritores controlados e não-controlados do $\mathrm{Me}$ dical Subject Headings (MeSH) e do Descritores em Ciências da Saúde (DeCS), complementando com as palavras-chave "brasileiro", "brasileiros", "Brazilian", "Brazilians", "Estudante de Psicologia", "Aluno de Psicologia", "Alunos de Psicologia", "Psychology Student", "Student, Psychology", "Psychology Students", "Students, Psychology", "Dentistry Students", "Student, Dentistry", "Nurse Students", "Student, Nurse", "Medicine Students", "Student, Medicine", para todas as bases de dados pesquisadas juntamente com os termos booleanos junto aos descritores AND, OR e NOT ${ }^{19}$. As buscas foram realizadas de forma separada para cada um dos cursos porque a concentração de todos os descritores resultava em nenhum trabalho encontrado. Os descritores e as palavras-chave para cada item da mnemônica estão descritos na Tabela 1.

Tabela 1: Descritores utilizados

\begin{tabular}{|c|c|c|c|}
\hline $\begin{array}{l}\text { Estratégias } \\
\text { PCC }\end{array}$ & Descritores controlados & Descritores não-controlados & Palavras-Chave \\
\hline \multirow[b]{2}{*}{ População } & $\begin{array}{l}\text { "Estudantes de Medi- } \\
\text { cina" }\end{array}$ & $\begin{array}{c}\text { “Aluno Medicina” OR “Alunos Medicina” OR "Estudante } \\
\text { Medicina” }\end{array}$ & \\
\hline & "Students, Medical" & $\begin{array}{c}\text { "Medical Students" OR "Student, Medical" OR "Medical } \\
\text { Student" }\end{array}$ & $\begin{array}{l}\text { "Medicine Students" } \\
\text { OR "Student, Medi- } \\
\text { cine" }\end{array}$ \\
\hline \multirow{2}{*}{ População 2} & $\begin{array}{l}\text { "Estudantes de Enferma- } \\
\text { gem" }\end{array}$ & $\begin{array}{c}\text { "Enfermeiras Estudantes" OR "Alunos de Enfermagem" } \\
\text { OR "Estudante de Enfermagem" OR "Enfermeiros Estu- } \\
\text { dantes" }\end{array}$ & \\
\hline & "Students, Nursing" & $\begin{array}{l}\text { "Pupil Nurses" OR “Student, Nursing” OR "Nurses, Pupil” } \\
\text { OR "Nurse, Pupil” OR "Pupil Nurse" OR "Nursing Stu- } \\
\text { dent" OR "Nursing Students" }\end{array}$ & $\begin{array}{l}\text { "Nurse Students" OR } \\
\text { "Student, Nurse" }\end{array}$ \\
\hline \multirow[b]{2}{*}{ População 3} & $\begin{array}{l}\text { "Estudantes de } \\
\text { Odontologia" }\end{array}$ & $\begin{array}{c}\text { "Alunos de Odontologia" OR "Aluno de Odontologia" OR } \\
\text { "Estudantes de Odontologia" }\end{array}$ & \\
\hline & "Students, Dental" & $\begin{array}{c}\text { "Dental Students" OR "Student, Dental" OR "Dental } \\
\text { Student" }\end{array}$ & $\begin{array}{l}\text { "Dentistry Students" } \\
\text { OR "Student, Den- } \\
\text { tistry" }\end{array}$ \\
\hline
\end{tabular}




\begin{tabular}{|c|c|c|c|}
\hline \multirow[b]{2}{*}{ População 4} & $\begin{array}{c}\text { "Estudantes de Ciências } \\
\text { da Saúde" }\end{array}$ & $\begin{array}{c}\text { "Estudante de Ciências da Saúde" OR "Aluno de Ciências } \\
\text { da Saúde" OR "Alunos de Ciências da Saúde" }\end{array}$ & \\
\hline & $\begin{array}{l}\text { "Students, Health Occu- } \\
\text { pations" }\end{array}$ & $\begin{array}{l}\text { "Health Occupations Students" OR "Health Occupations } \\
\text { Student" OR "Occupations Student, Health" OR "Occupa- } \\
\text { tions Students, Health" OR "Student, Health Occupations" }\end{array}$ & \\
\hline \multirow[b]{2}{*}{ População 5} & $\begin{array}{l}\text { "Estudantes de Saúde } \\
\text { Pública" }\end{array}$ & $\begin{array}{l}\text { "Estudante de Saúde Pública” OR “Aluno de Saúde } \\
\text { Pública” OR “Alunos de Saúde Pública” }\end{array}$ & \\
\hline & "Students, Public Health" & $\begin{array}{l}\text { "Health Student, Public" OR "Health Students, Public" OR } \\
\text { "Public Health Student" OR "Student, Public Health" OR } \\
\text { "Public Health Students" }\end{array}$ & \\
\hline \multirow[b]{2}{*}{ População 6} & Psicologia & "Curso de Psicologia" & $\begin{array}{l}\text { "Estudante de Psico- } \\
\text { logia" OR "Aluno de } \\
\text { Psicologia" OR “Alu- } \\
\text { nos de Psicologia" }\end{array}$ \\
\hline & Psychology & $\begin{array}{l}\text { "Side Effects, Psychological" OR "Psychological Side } \\
\text { Effects" OR "Psychological Side Effect" OR "Side Effect, } \\
\text { Psychological” OR "Psychosocial Factors" OR "Factor, } \\
\text { Psychosocial" OR "Factors, Psychosocial" OR "Psychoso- } \\
\text { cial Factor" OR "Psychological Factors" OR "Factors, Psy- } \\
\text { chological" OR "Factor, Psychological” OR "Psychological } \\
\text { Factor" OR "Psychologists" OR "Psychologist" }\end{array}$ & $\begin{array}{l}\text { "Psychology Student" } \\
\text { OR "Student, Psychol- } \\
\text { ogy" OR "Psychology } \\
\text { Students" OR "Stu- } \\
\text { dents, Psychology" }\end{array}$ \\
\hline \multirow[t]{2}{*}{ Conceito } & $\begin{array}{l}\text { "Esgotamento } \\
\text { Profissional" }\end{array}$ & $\begin{array}{c}\text { Burnout OR "Desgaste Profissional” OR "Estresse Ocupa- } \\
\text { cional" OR "Estafa Profissional” OR "Estresse Profissio- } \\
\text { nal” OR "Esgotamento Psíquico" OR "Esgotamento Emo- } \\
\text { cional” OR "Exaustão Emocional e Física” OR "Exaustão } \\
\text { Profissional” }\end{array}$ & \\
\hline & "Burnout, Professional" & $\begin{array}{c}\text { "Professional Burnout" OR “Occupational Burnout" OR } \\
\text { "Burnout, Occupational" }\end{array}$ & Burnout \\
\hline \multirow{2}{*}{ Contexto } & Brasil & & $\begin{array}{l}\text { Brasileiro OR } \\
\text { Brasileiros }\end{array}$ \\
\hline & Brazil & & $\begin{array}{l}\text { Brazilian OR } \\
\text { Brazilians }\end{array}$ \\
\hline
\end{tabular}

O levantamento bibliográfico foi realizado por pares de pesquisadores durante o mês de janeiro de 2018, guiado por um roteiro elaborado pelos autores com os critérios de elegibilidade: artigos completos disponíveis; artigos disponíveis nos idiomas português, inglês ou espanhol; artigos completos que respondessem à pergunta norteadora do estudo. Para auxiliar a leitura exaustiva dos títulos, resumos e conteúdo, foi utilizado o software Start. Com relação ao recorte temporal, optou-se por utilizar todo o período disponível nas bases para obtenção de uma quantidade maior de trabalhos.

A busca inicial resultou em um total de 309 trabalhos. Após a aplicação dos critérios de elegibilidade, a amostra final foi composta de 16 manuscritos. O processo de seleção dos e o resultado final da revisão encontram-se descritos na Figura 1. A quantidade de trabalhos iniciais, trabalhos duplicados e trabalhos finais por base de dados, está descrita na Tabela 2. 
Figura 1:Resultado Final da Revisão

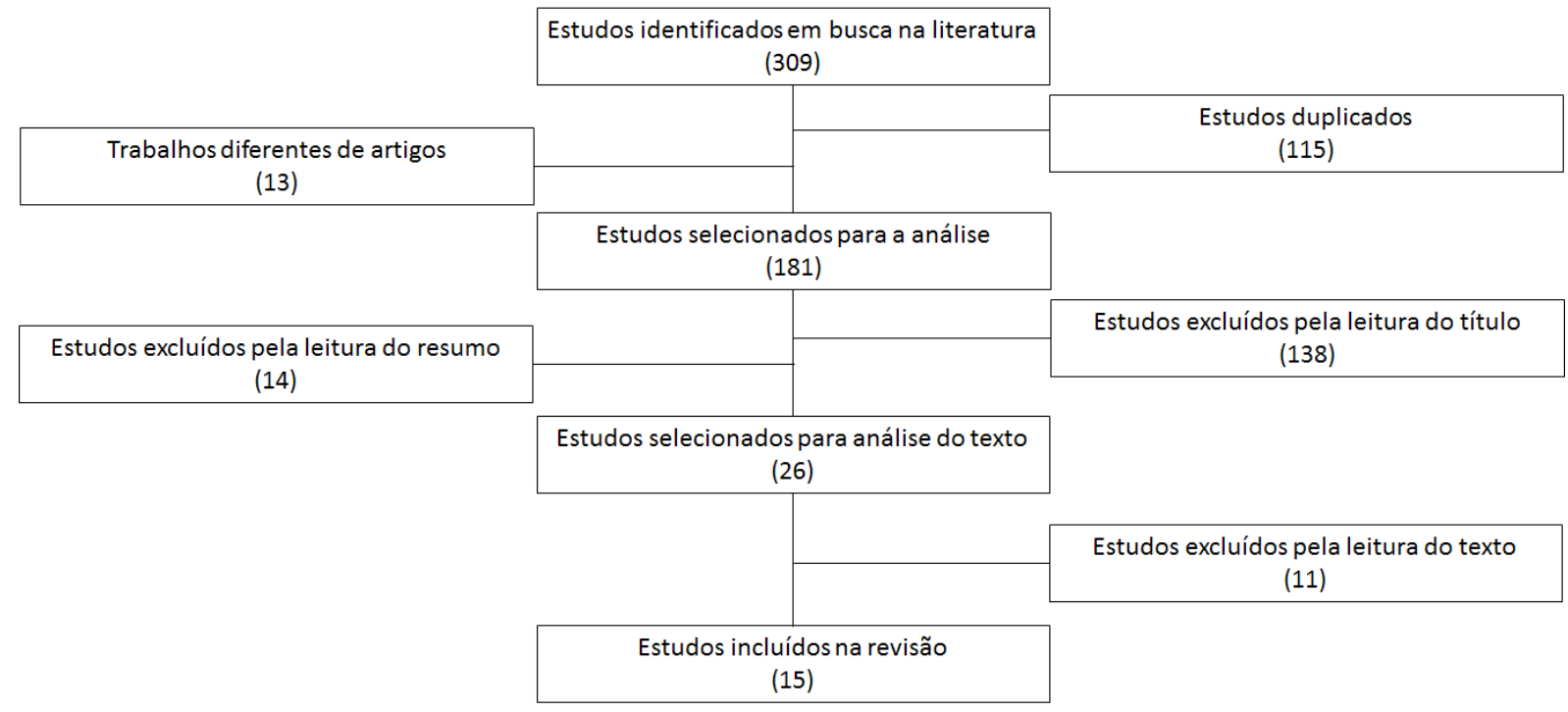

Tabela 2: Total de estudos por base de dados

\begin{tabular}{cccc}
\hline Base de dados & $\begin{array}{c}\text { Amostra } \\
\text { Inicial }\end{array}$ & $\begin{array}{c}\text { Estudos duplica- } \\
\text { dos }\end{array}$ & Amostra Final \\
\hline Cochrane & 1 & 0 & 0 \\
Lilacs & 74 & 10 & 3 \\
\hline PubMed & 129 & 72 & 5 \\
Scielo & 27 & 9 & 5 \\
\hline Scopus & 58 & 15 & 2 \\
Web of Science & 20 & 9 & 0 \\
\hline Total de estudos & 309 & 115 & 15 \\
\hline
\end{tabular}

\section{Resultados}

A aplicação dos critérios de elegibilidade possibilitou a seleção final de quinze estudos. Do total, oito estudos analisaram alunos do curso de medicina ${ }^{20-27}$, cinco estudos analisaram alunos do curso de enfermagem ${ }^{28-32}$ e dois estudos analisaram os alunos do curso de odontologia ${ }^{33,34}$. Na Tabela 3, é possível identificar os estudos analisados, os autores, o Estado brasileiro onde o estudo foi realizado, o objetivo, o curso de graduação estudado, o questionário utilizado para a coleta de dados, os resultados obtidos, o ano de publicação do artigo e a prevalência de ocorrência entre os sexos. 
Tabela 3: Resumo da busca

\begin{tabular}{|c|c|c|c|c|c|c|c|c|}
\hline Título & Autores & Local & Objetivo & Área & Instrumento & Resultados & Ano & Ocorrência \\
\hline $\begin{array}{l}\text { Trans- } \\
\text { tornos } \\
\text { emocionais } \\
\text { e a for- } \\
\text { mação em } \\
\text { Medicina: } \\
\text { um estudo } \\
\text { longitudi- } \\
\text { nal }\end{array}$ & $\begin{array}{l}\text { Benevi- } \\
\text { des-Perei- } \\
\text { ra AMT, } \\
\text { Gonçal- } \\
\text { ves MB. }\end{array}$ & PR & $\begin{array}{l}\text { Avaliar alunos } \\
\text { de um curso de } \\
\text { Medicina de } \\
\text { uma universida- } \\
\text { de do interior do } \\
\text { Paraná, acom- } \\
\text { panhando-os } \\
\text { do primeiro ao } \\
\text { último ano. }\end{array}$ & Medicina & MBI & $\begin{array}{l}\text { Transtornos emocionais foram maio- } \\
\text { res no terceiro e quarto ano do curso } \\
\text { com aumento gradativo do Burnout } \\
\text { até o quarto ano para depois decair. }\end{array}$ & 2009 & Igual \\
\hline $\begin{array}{l}\text { Burnout } \\
\text { Syndrome } \\
\text { and associ- } \\
\text { ated factors } \\
\text { among } \\
\text { medical } \\
\text { students: a } \\
\text { cross-sec- } \\
\text { tional study }\end{array}$ & $\begin{array}{c}\text { Costa } \\
\text { EFO, } \\
\text { Santos } \\
\text { SA, } \\
\text { Santos } \\
\text { ATRA, } \\
\text { Melo EV, } \\
\text { Andrade } \\
\text { TM. }\end{array}$ & SE & $\begin{array}{l}\text { Avaliar a preva- } \\
\text { lência e os níveis } \\
\text { de síndrome de } \\
\text { burnout entre } \\
\text { estudantes de } \\
\text { medicina da } \\
\text { Universidade } \\
\text { Federal de } \\
\text { Sergipe-Brasil e } \\
\text { identificar fato- } \\
\text { res associados. }\end{array}$ & Medicina & MBI-SS & $\begin{array}{l}\text { A prevalência de Burnout foi de } \\
10,3 \% \text {, sendo maior entre aquele que } \\
\text { se sentiam inseguros, aqueles que se } \\
\text { sentiam desconfortáveis com o curso } \\
\text { e aqueles que não viam o curso como } \\
\text { fonte de prazer. }\end{array}$ & 2012 & Homem \\
\hline $\begin{array}{c}\text { Burnout en } \\
\text { estudiantes } \\
\text { de odon- } \\
\text { tología: } \\
\text { evaluación } \\
\text { a través } \\
\text { mbi - versi- } \\
\text { ón estu- } \\
\text { diantes }\end{array}$ & $\begin{array}{c}\text { Garbin } \\
\text { ASC, } \\
\text { Saliba } \\
\text { AN, San- } \\
\text { tos RR, } \\
\text { Prado RL, } \\
\text { Garbin } \\
\text { AJI. }\end{array}$ & SP & $\begin{array}{c}\text { Determinar a } \\
\text { presença e o } \\
\text { nível de Síndro- } \\
\text { me de Burnout } \\
\text { nos estudantes, } \\
\text { comparando o } \\
\text { início e o final } \\
\text { da graduação } \\
\text { e verificando a } \\
\text { correlação entre } \\
\text { as sub-escalas } \\
\text { e o conjunto de } \\
\text { variáveis socio- } \\
\text { demográficas. }\end{array}$ & $\begin{array}{l}\text { Odonto- } \\
\text { logia }\end{array}$ & MBI-SS & $\begin{array}{c}\text { Houve correlação entre exaustão } \\
\text { emocional ( }<0,01) \text {, descrença }(\mathrm{p} \\
<0,05) \text {, eficácia profissional }(\mathrm{p}<0,01) \\
\text { e o ano em que o aluno está matricu- } \\
\text { lado no curso. }\end{array}$ & 2012 & Igual \\
\hline
\end{tabular}

\begin{tabular}{|c|c|c|c|c|c|c|c|c|}
\hline $\begin{array}{c}\text { Manifes- } \\
\text { tações da } \\
\text { síndrome } \\
\text { de burnout } \\
\text { entre estu- } \\
\text { dantes de } \\
\text { graduação } \\
\text { em enfer- } \\
\text { magem }\end{array}$ & $\begin{array}{c}\text { Tomas- } \\
\text { chewski- } \\
\text {-Barlem } \\
\text { JG, } \\
\text { Lunardi } \\
\text { VL, Ra- } \\
\text { mos AM, } \\
\text { Silveira } \\
\text { RS, Bar- } \\
\text { lem ELD, } \\
\text { Ernandes } \\
\text { CM. }\end{array}$ & Sul do Brasil & $\begin{array}{l}\text { Conhecer as } \\
\text { manifestações da } \\
\text { síndrome de bur- } \\
\text { nout presentes } \\
\text { entre estudantes } \\
\text { de graduação em } \\
\text { enfermagem. }\end{array}$ & $\begin{array}{c}\text { Enferma- } \\
\text { gem }\end{array}$ & $\begin{array}{c}\text { Análise } \\
\text { Textual } \\
\text { Discursiva }\end{array}$ & $\begin{array}{l}\text { Os estudantes não manifestaram } \\
\text { necessariamente características } \\
\text { específicas a todas as dimensões da } \\
\text { síndrome, apresentando maior ênfase } \\
\text { em uma ou em outra dimensão. }\end{array}$ & 2013 & $\begin{array}{l}\text { Sem infor- } \\
\text { mação }\end{array}$ \\
\hline $\begin{array}{l}\text { Burnout } \\
\text { and career } \\
\text { choice } \\
\text { motivation } \\
\text { in medical } \\
\text { students }\end{array}$ & $\begin{array}{l}\text { Pagnin } \\
\text { D, De } \\
\text { Queiroz } \\
\text { V, Olivei- } \\
\text { ra Filho } \\
\text { MAD, } \\
\text { Gonzalez } \\
\text { NVA, } \\
\text { Salgado } \\
\text { AET, } \\
\text { Oliveira } \\
\text { BCE, } \\
\text { Lodi CS, } \\
\text { Melo }\end{array}$ & RJ & $\begin{array}{l}\text { Investigar a in- } \\
\text { fluência da moti- } \\
\text { vação da escolha } \\
\text { de carreira no } \\
\text { risco de burnout } \\
\text { em estudantes de } \\
\text { medicina. }\end{array}$ & Medicina & MBI-SS & $\begin{array}{l}\text { Os estudantes de medicina motiva- } \\
\text { dos por doença pessoal ou doença } \\
\text { ou morte de membros da família } \\
\text { revelaram um maior Burnout quando } \\
\text { comparado com os alunos com outras } \\
\text { motivações. }\end{array}$ & 2013 & Mulher \\
\hline
\end{tabular}




\begin{tabular}{|c|c|c|c|c|c|c|c|c|}
\hline $\begin{array}{c}\text { (Des) } \\
\text { conheci- } \\
\text { mento da } \\
\text { Síndrome } \\
\text { de Bur- } \\
\text { nout entre } \\
\text { acadêmicos } \\
\text { de enfer- } \\
\text { magem }\end{array}$ & $\begin{array}{l}\text { Corral- } \\
\text {-Mulato } \\
\text { S, Bueno } \\
\text { SMV. }\end{array}$ & SP & $\begin{array}{l}\text { Investigar o } \\
\text { conhecimento } \\
\text { do aluno de } \\
\text { enfermagem } \\
\text { sobre a Síndro- } \\
\text { me de Burnout } \\
\text { e promover } \\
\text { um programa } \\
\text { educativo nesse } \\
\text { sentido. }\end{array}$ & $\begin{array}{l}\text { Enferma- } \\
\text { gem }\end{array}$ & - & $\begin{array}{c}\text { Ao todo, } 31 \% \text { não conheciam a sín- } \\
\text { drome, } 65 \% \text { conheciam, porém, } \\
21 \% \text { não se lembravam e } 315,8 \% \text { se } \\
\text { equivocaram. }\end{array}$ & 2014 & - \\
\hline $\begin{array}{l}\text { Evidência } \\
\text { de validade } \\
\text { prelimi- } \\
\text { nar da } \\
\text { escala de } \\
\text { depressão } \\
\text { (EDEP): } \\
\text { um estudo } \\
\text { com alunos } \\
\text { de enfer- } \\
\text { magem }\end{array}$ & $\begin{array}{l}\text { Rueda } \\
\text { FJM, Al- } \\
\text { ves SMM, } \\
\text { Baptista } \\
\text { MN. }\end{array}$ & SP & $\begin{array}{l}\text { Buscar evidên- } \\
\text { cias baseando-se } \\
\text { na relação, com } \\
\text { outras variáveis, } \\
\text { de uma versão } \\
\text { de } 32 \text { itens da } \\
\text { EDEP, corre- } \\
\text { lacionando-as } \\
\text { com a Escala de } \\
\text { Vulnerabilidade } \\
\text { ao Estresse } \\
\text { no Trabalho } \\
\text { (EVENT) e a } \\
\text { Maslach Burnout } \\
\text { Inventory - } \\
\text { Human Services } \\
\text { Survey (MBI- } \\
\text {-HSS). }\end{array}$ & $\begin{array}{l}\text { Enferma- } \\
\text { gem }\end{array}$ & MBI-SS & $\begin{array}{l}\text { Os alunos com Burnout corresponde- } \\
\text { ram a } 11 \% \text { da amostra. }\end{array}$ & 2014 & $\begin{array}{l}\text { Sem infor- } \\
\text { mação }\end{array}$ \\
\hline $\begin{array}{l}\text { Burnout } \\
\text { syndrome } \\
\text { among un- } \\
\text { dergraduate } \\
\text { nursing } \\
\text { students } \\
\text { at a public } \\
\text { university }\end{array}$ & $\begin{array}{c}\text { Tomas- } \\
\text { chewski- } \\
\text {-Barlem } \\
\text { JG, Lu- } \\
\text { nardi VL, } \\
\text { Lunardi } \\
\text { GL, Bar- } \\
\text { lem ELD, } \\
\text { Silveira } \\
\text { RS, Vidal } \\
\text { DAS. }\end{array}$ & Sul do Brasil & $\begin{array}{c}\text { Investigar } \\
\text { a síndrome } \\
\text { de burnout e } \\
\text { sua relação } \\
\text { com variáveis } \\
\text { demográficas } \\
\text { e acadêmicas } \\
\text { entre estudantes } \\
\text { de graduação de } \\
\text { enfermagem de } \\
\text { uma universida- } \\
\text { de pública do sul } \\
\text { do Brasil. }\end{array}$ & $\begin{array}{l}\text { Enferma- } \\
\text { gem }\end{array}$ & MBI-SS & $\begin{array}{c}\text { Foi descoberto que os estudantes não } \\
\text { têm a Síndrome de Burnout. }\end{array}$ & 2014 & $\begin{array}{l}\text { Sem infor- } \\
\text { mação }\end{array}$ \\
\hline
\end{tabular}

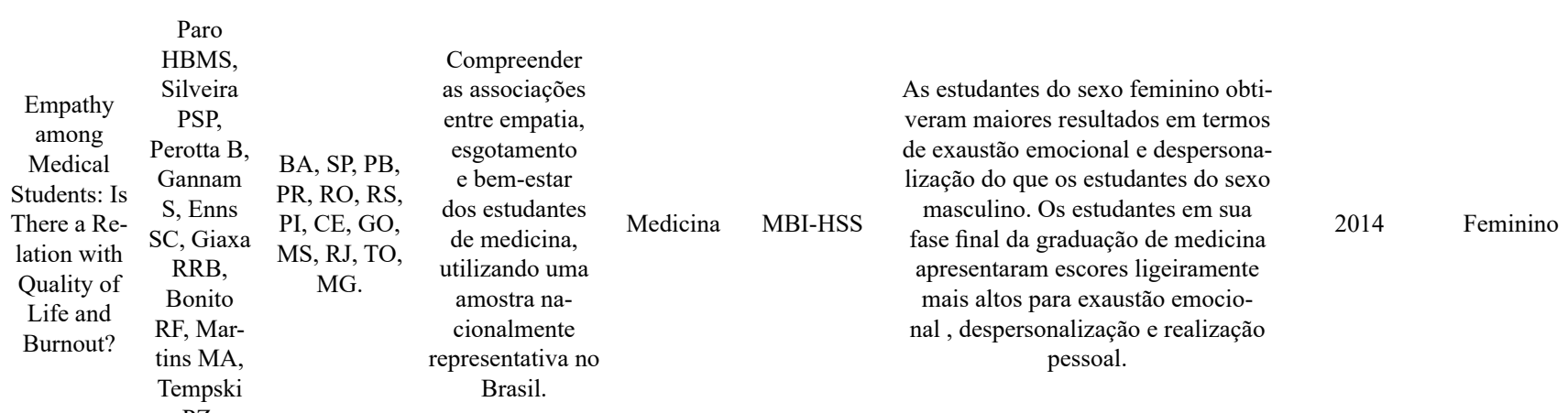




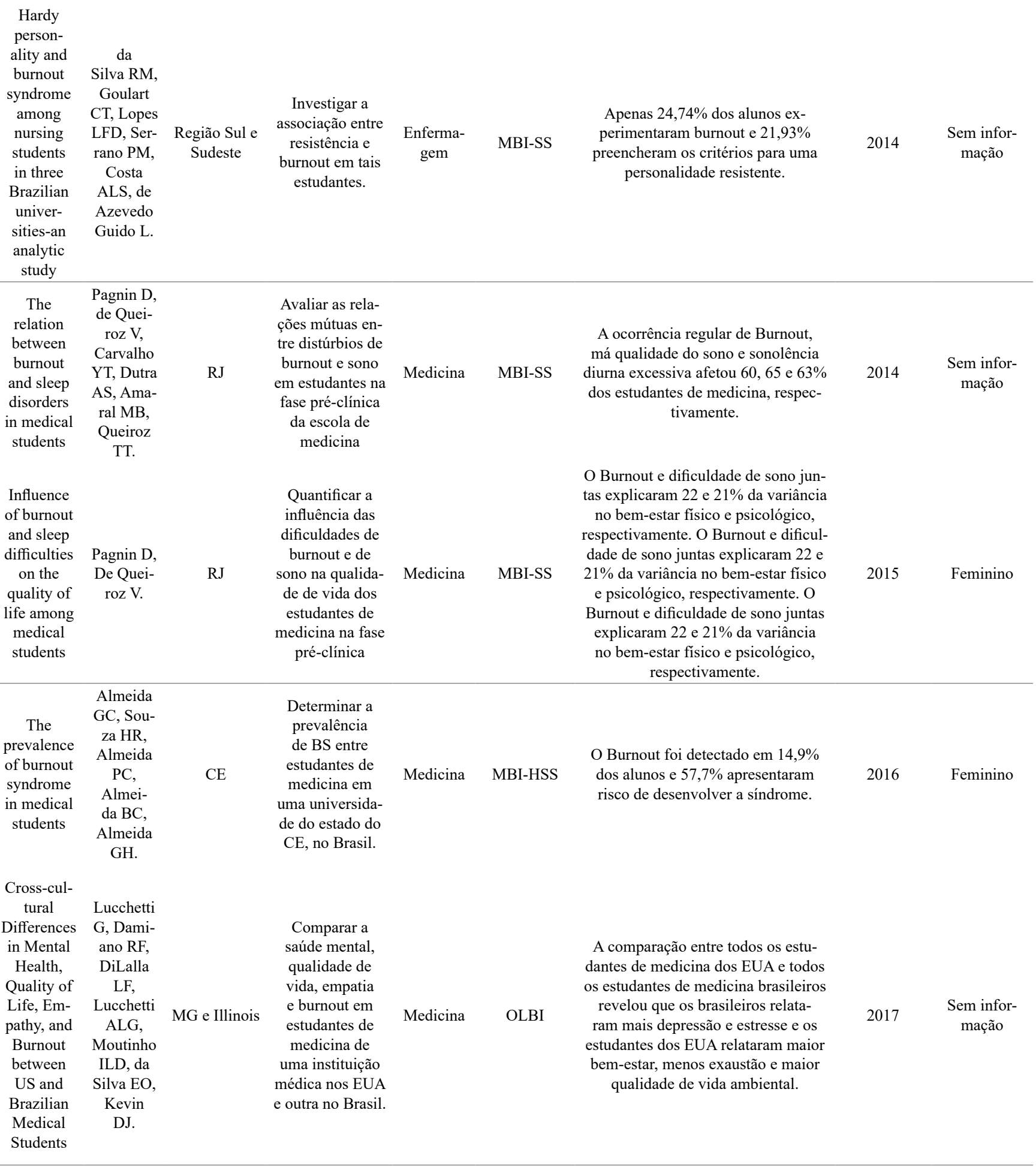




\begin{tabular}{|c|c|c|c|c|c|c|c|}
\hline $\begin{array}{l}\text { Chang- } \\
\text { es in } \\
\text { salivary } \\
\text { microbiota } \\
\text { increase } \\
\text { volatile } \\
\text { sulfur } \\
\text { compounds } \\
\text { production } \\
\text { in healthy } \\
\text { male sub- } \\
\text { jects with } \\
\text { academ- } \\
\text { ic-related } \\
\text { chronic } \\
\text { stress }\end{array}$ & $\begin{array}{l}\text { Nani } \\
\text { BD, } \\
\text { Lima PO, } \\
\text { Marcon- } \\
\text { des FK, } \\
\text { Groppo } \\
\text { FC, Ro- } \\
\text { lim GS, } \\
\text { Moraes } \\
\text { AB, Co- } \\
\text { go-Muller } \\
\text { K, Franz- } \\
\text {-Montan } \\
\text { M. }\end{array}$ & SP & $\begin{array}{c}\text { Investigar } \\
\text { as associações } \\
\text { entre bacté- } \\
\text { rias salivares, } \\
\text { emanações orais } \\
\text { de compostos de } \\
\text { enxofre voláteis } \\
\text { e estresse crôni- } \\
\text { co relacionado } \\
\text { ao acadêmico } \\
\text { em indivíduos } \\
\text { saudáveis do } \\
\text { sexo masculino. }\end{array}$ & $\begin{array}{l}\text { Odon- } \\
\text { tologia }\end{array}$ & MBI-SS & $\begin{array}{l}\text { O grupo de estudantes estres- } \\
\text { sados apresentou emanações orais } \\
\text { aumentadas de sulfeto de hidrogênio } \\
\text { e sulfeto de dimetilo, juntamente } \\
\text { com níveis de Solobacterium moorei } \\
\text { salivares mais elevados. }\end{array}$ & 2017 \\
\hline
\end{tabular}

Fonte: dados da pesquisa

Os estudos analisando alunos de medicina foram realizados em quatorze estados brasileiros diferentes medicina20-26 e em um Estado dos EUA em um estudo comparativo27. As análises variaram com seis estudos com análises restritas a estudantes de uma única universidade20-23, 25, 26, um estudo multicêntrico em treze Estados brasileiros 24 e um estudo comparativo entre estudantes de MG e de Illinois (EUA)27. O Estado brasileiro mais estudado foi o Rio de Janeiro com quatro trabalhos, incluindo o multicêntrico, sendo três pesquisas realizadas na Universidade Federal Fluminense22, 23, 25.

Os estudos analisando estudantes de enfermagem foram realizados no Estado de São Paulo29, 30 e nas regiões Sul e Sudeste sem indicação dos Estados analisados28, 31, 32. Semelhante aos estudos em medicina, as análises ficaram concentradas em estudantes de apenas uma universidade com quatro estudos28-31 e um estudo analisando três instituições simultaneamente32. Entretanto, diferentemente dos estudos sobre alunos de medicina, as cidades e os nomes das instituições com os cursos de enfermagem ficaram sem identificação detalhada nos trabalhos sobre estudantes de enfermagem.

Os estudos analisando estudantes de odontologia foram realizados somente no Estado de São Paulo e nas cidades de Araçatuba33 e Piracicaba34. Da mesma forma que ocorreu com os estudos sobre estudantes de medicina e enfermagem, os trabalhos sobre odontologia também foram concentrados em apenas alunos de uma única instituição. As instituições que tiveram estudantes analisados foram a Universidade Estadual Paulista33 e Universidade Estadual de Campinas34.

Com relação aos objetivos dos trabalhos, observou-se diversidade nos estudos sobre estudantes de medicina quanto à forma de abordagem do Burnout. Apenas dois trabalhos tinham como objetivo determinar a prevalência da síndrome na população estudada21, 26. Quanto aos objetivos dos demais trabalhos, três avaliaram o Burnout com outros fatores emocionais $20,24,27$, dois investigaram a Síndrome de Burnout com distúrbios do sono23, 25 e um estudo investigou o grau de influência da escolha da carreira médica com a propensão do surgimento do Burnout22.

Sobre os objetivos dos estudos de enfermagem, observou-se uma abordagem mais investigativa por parte dos autores. Ao todo, dois trabalhos buscaram investigar associações e relações da Síndrome de Burnout com outras variáveis31, 32, um estudo buscou conhecer a manifestação da síndrome nos estudantes 28 e um trabalhou objetivou investigar o conhecimento dos estudantes sobre o Burnout29. Apenas um trabalho teve como objetivo algo diferente de uma investigação ao buscar evidências na relação da Escala de Depressão (EDEP) com a Escala de Vulnerabilidade ao Estresse no Trabalho (EVENT) e a Maslach Burnout Inventory - Human Services Survey (MBI-HSS) 30 .

Os estudos sobre odontologia apresentaram objetivos distintos associados a Síndrome de Burnout. O estudo de Garbin33 teve como objetivo determinar a presença da síndrome nos estudantes e comparar com o início e o fim do curso. Já o 
estudo de Nani34 buscou investigar as associações entre bactérias salivares, emanações orais de compostos de enxofre e Burnout em indivíduos do sexo masculino, sendo o único estudo encontrado que limitou a população pelo gênero do estudante.

Com relação aos questionários utilizados para a mensuração da Síndrome de Burnout, houve prevalência pela utilização do Maslach Burnout Inventory - Student Survey (MBI-SS) com a utilização em nove dos quinze trabalhos21-23, 25, 30-34. O segundo questionário mais utilizado foi o Maslach Burnout Inventory - Health Service Suvey (MBI-HSS), sendo utilizado duas vezes24 26. Os outros trabalhos utilizaram o Maslach Burnout Inventory (MBI)20, o Oldenburg Burnout Inventory (OLBI)27 e uma análise textual discursiva28.

Sobre os resultados dos estudos que analisaram estudantes de medicina, o estudo comparativo entre MG e Illinois (EUA) revelou maior Burnout para estudantes brasileiros sem realizar discriminação quanto ao sexo dos alunos27. Os estudantes que optaram pelo curso de medicina por conta de motivações provocadas por doença pessoal ou familiar ou morte de familiares apresentaram maior propensão de desenvolvimento da síndrome com maior incidência em alunos do sexo feminino 22 . Com relação ao desenvolvimento do Burnout durante o período de graduação, o estudo de Benevides-Pereira e Gonçalves20 aponta ocorrência igual em ambos os sexos e um aumento gradativo da Síndrome de Burnout até o quarto ano de graduação para depois decair, porém, o estudo de Paro et al24 aponta ocorrência maior e estudantes do sexo feminino e que o Burnout é ligeiramente mais elevado nos estudantes na fase final do curso.

Os estudos relacionando Síndrome de Burnout e disfunções do sono são possivelmente trabalhos derivados da mesma pesquisa por conta dos autores, objetivos e instituição participantes, onde $60 \%$ dos alunos foram afetados pela síndrome com predominância de ocorrência entre estudantes do sexo feminino23 25. Apesar do número elevado, o estudo realizado em Sergipe apresentou prevalência de Burnout em 10,3\% com ocorrência maior em estudantes do sexo masculino21. Já o estudo feito no Ceará indicou prevalência em 14,9\% dos estudantes com predominância entre o sexo feminino 25.

Sobre os resultados dos estudos que analisaram estudantes de enfermagem, apenas o estu- do de Corral-Mulato e Bueno29 abordou o conhecimento dos estudantes sobre a Síndrome de Burnout sem mensurar a ocorrência nos alunos, sendo conhecida por $65 \%$ da amostra. Dois estudos apresentaram a ocorrência de Burnout em $11 \% 30$ e $24,74 \% 32$, enquanto outros dois estudos indicaram que os estudantes analisados não apresentavam a síndrome28, 30, porém, é possível que ambos sejam trabalhos derivados da mesma pesquisa por conta dos autores, objetivos e instituição participantes. Com relação à prevalência entre os sexos dos estudantes, nenhum estudo informou em qual sexo a ocorrência foi maior.

Sobre os resultados dos estudos que analisaram estudantes de odontologia, existem indícios de correlação entre a Síndrome de Burnout e o ano de graduação do estudante com maior ocorrência em estágios pré-clínicos, ocorrendo de forma equivalente entre ambos os sexos33. Os estudantes do sexo masculino que apresentaram maiores níveis de Burnout possuíram emanações orais aumentadas de sulfeto de hidrogênio e sulfeto de dimetilo, juntamente com níveis de Solobacterium moorei salivares mais elevados34. Em ambos os estudos, a porcentagem de ocorrência da síndrome entre os alunos foi omitida pelos pesquisadores.

\section{Discussão}

A análise dos resultados possibilitou o levantamento de pontos pertinentes sobre as pesquisas envolvendo Síndrome de Burnout em estudantes de graduação. $\mathrm{O}$ curso de medicina apresentou predominância na quantidade de estudos com oito estudos, seguido pelo curso de enfermagem com cinco estudos e odontologia com dois. Nenhum estudo foi encontrado para o curso de psicologia apesar de ter sido a busca com a maior utilização de descritores.

Os estudos sobre alunos do curso de medicina foram os trabalhos que apresentaram a maior abrangência temporal de publicação: de 2009 a 2017. Apesar da ocorrência de um hiato entre os anos de 2009 e 2013, o interesse dos pesquisadores pelo assunto apresenta uma constância temporal, diferentemente do ocorrido com os estudos sobre os estudantes de enfermagem onde estão todos concentrados entre os anos de 2013 e 2014. O in- 
teresse com relação aos estudantes de odontologia apresentou-se muito pontual com publicações em apenas dois anos e com intervalo de cinco anos entre as duas.

Além da concentração em dois anos, as pesquisas sobre estudantes de graduação do curso de enfermagem foram todos realizadas nas Regiões Sul e Sudeste, sendo o Estado de São Paulo o único Estado discriminado com dois estudos realizados. A mesma concentração no Estado de São Paulo também ocorre com os trabalhos envolvendo os alunos do curso de odontologia. Entretanto, os estudos sobre os alunos do curso de medicina apresentaram padrão completamente diferente, sendo realizado em vários Estados brasileiros e nenhum trabalho analisando unicamente uma instituição paulista.

A concentração também ocorre com alguns grupos de autores das pesquisas: três estudos sobre estudantes do curso de medicina apresentaram composição autoral semelhante e dois trabalhos sobre o curso de enfermagem apresentaram autorias análogas. Apesar de aparentar se tratar de grupos especializados sobre a Síndrome de Burnout, a proximidade dos anos de publicação dos estudos indicam que os artigos são apresentações de resultados provenientes de uma única pesquisa desdobrada em diversas publicações. O desdobramento de uma pesquisa em várias publicações pode significar que vários artigos publicados utilizaram a mesma amostra de análise, replicando um resultado padrão.

Apesar dos resultados dos estudos apontarem que os estudantes do curso de enfermagem ser o único grupo com pesquisas indicando a não manifestação da Síndrome de Burnout, é equivocado aferir que os alunos de enfermagem são menos propensos a manifestação da síndrome. Os estudos que apresentaram tais resultados são de autoria de um grupo de autores semelhantes o que pode indicar uma amostra igual ou com características parecidas. Entretanto, é destacável o resultado negativo para a manifestação da Síndrome de Burnout para aquele grupo específico de estudantes.

Tais padrões observados com as publicações salientam uma carência de estudos sobre a Síndrome de Burnout em estudantes de graduação dos cursos de saúde no Brasil além de dificultarem a realização de um diagnóstico mais assertivo sobre o problema. Os padrões acerca das publicações podem ser expli- cados por ser um assunto de interesse recente onde doze dos quinze estudos foram publicados a partir do ano de 2013. A carência de estudos sobre o assunto aponta para oportunidades para a realização de diversos estudos abordando o tema em questão.

\section{Conclusão}

O estudo encontrou quinze trabalhos que abordaram a Síndrome de Burnout em estudantes de graduação dos cursos de medicina, enfermagem e odontologia em instituições brasileiras, sendo oito trabalhos com estudantes de medicina, cinco com estudantes de enfermagem, dois com estudantes de odontologia e nenhum trabalho com estudantes de psicologia. Em adição, apesar de ser um assunto de interesse desde 2009, foram observadas diversas carências e oportunidades para estudos futuros, principalmente na área da psicologia.

\section{Referências}

1. Secomb J. A systematic review of peer teaching and learning in clinical education. J Clin Nurs. 2008; 17 (6): 703-16.

2. Ladyshewsky R. A quasi-experimental study of the differences in performance and clinical reasoning using individual learning versus reciprocal peer coaching. Physiotherapy Theory and Practice. 2008;18 (1): 17-31.

3. Salmela-Aro K, Kiuru N, Leskinen E, Nurmi J-E. School burnout inventory (SBI) reliability and validity. European Journal of Psychological Assessment. 2009; 25 (1): 48-57.

4. Skodova Z, Lajciakova P. The effect of personality traits and psychosocial training on burnout syndrome among healthcare students. Nurse Education Today. 2013; 33 (11): 1311-1315.

5. Santos VEP, Radünz V. O estresse de acadêmicas de enfermagem e a segurança do paciente. Rev. enferm. 2011; 19 (4): 616-20.

6. Polman R, Borkoles E, Nicholls AR. Type D personality, stress, and symptoms of burnout: The influence of avoidance coping and social support. British Journal of Health Psychology. 2010; 15 (3): 681-696.

7. Dyrbye LN, Thomas MR, Harper W, Massie Jr. FS, Power DV, Eacker A, Szydlo DW, Novotny PJ, Sloan JA, Shanafelt TD. The learning environment and medical student burnout: A multicentre study. Medical Education. 2009; 43 (3): 274-282.

8. Nicola R, McNeeley MF, Bhargava P. Burnout in radiology. Current Problems in Diagnostic Radiology. 2015; 44 (5): 389-390. 
9. Maslach C, Schaufeli WB, Leiter MP. Job burnout. Annual Review of Psychology. 2001; 52 (1): 397-422.

10. Leiter MP, Maslach C. Latent burnout profiles: A new approach to understanding the burnout experience. Burnout Research. 2016; 3 (4): 89-100.

11. Batista KM, Bianchi ERF. Estresse do enfermeiro em unidade de emergência. Ver Latino-Am Enfermagem. 2006; 14: 534-9.

12. Alarcon G, Eschleman KJ, Bowling NA. Relationships between personality variables and burnout: A meta-analysis. Work and Stress. 2009; 23 (3): 244-263.

13. Maslach C, Jackson SE. The measurement of experienced burnout. Journal of Organizational Behavior. 1981; 2 (2): 99-113.

14. Kinser P, Braun S, Deeb G, Carrico C, Dow A. Awareness is the first step: An interprofessional course on mindfulness \& mindful-movement for healthcare professionals and students. Complementary Therapies in Clinical Practice. 2016; 25 (1): 18-25.

15. Volpe U, Luciano M, Palumbo C, Sampogna G, Del Vecchio V, Fiorillo A. Risk of burnout among early career mental health professionals. Journal of Psychiatric and Mental Health Nursing. 2014; 21 (9): 774-781.

16. Dyrbye LN, Thomas MR, Massie FS, Power DV, Eacker A, Harper W, Durning S, Moutier C, Szydlo DW, Novotny PJ, Sloan JA, Shanafelt TD. Burnout and suicidal ideation among U.S. medical students. Ann Intern Med. 2008; 5 (149): 334-341

17. Wolf MR, Rosenstock JB. Inadequate Sleep and Exercise Associated with Burnout and Depression among Medical Students. Academic Psychiatry. 2017; 2 (1): 174-179.

18. Joanna Briggs Institute - Methodology for JBI Scoping Reviews. https://reviewersmanual.joannabriggs.org/ display/MANUAL/Chapter+11\%3A+Scoping+reviews. Acessado em 18 de Janeiro de 2018.

19. Santos CMC, Pimenta CAM, Nobre MRC. A estratégia PICO para construção da pergunta de pesquisa e a busca de evidências. Revista Latino-Americana de Enfermagem. 2007; 15 (3): 508-511.

20. Benevides-Pereira AMT, Gonçalves MB. Transtornos emocionais e a formação em Medicina: um estudo longitudinal. Revista Brasileira de Educação Médica. 2009; 33 (1): 10-23.

21. Costa EFO, Santos SA, Santos ATRA, Melo EV, Andrade TM. Burnout Syndrome and associated factors among medical students: a cross-sectional study. Clinics. 2012; 67 (6): 573-579.

22. Pagnin D, De Queiroz V, Oliveira Filho MAD, Gonzalez NVA, Salgado AET, Oliveira BCE, Lodi CS, Melo RMDS. Burnout and career choice motivation in medical students. Medical Teaching. 2013; 35(5):388-94.
23. Pagnin D, de Queiroz V, Carvalho YT, Dutra AS, Amaral MB, Queiroz TT. The relation between burnout and sleep disorders in medical students. Academic Psychiatry. $2014 ; 38(4): 438-44$.

24. Paro HBMS, Silveira PSP, Perotta B, Gannam S, Enns SC, Giaxa RRB, Bonito RF, Martins MA, Tempski PZ. Empathy among Medical Students: Is There a Relation with Quality of Life and Burnout? PLoS ONE. 2014; 9(4): e94133.

25. Pagnin D, De Queiroz V. Influence of burnout and sleep difficulties on the quality of life among medical students. Springerplus. 2015; 4: 676.

26. Almeida GC, Souza HR, Almeida PC, Almeida BC, Almeida GH. The prevalence of burnout syndrome in medical students. Archives Clinical Psychiatry. 2016; 43 (1):6-10.

27. Lucchetti G, Damiano RF, DiLalla LF, Lucchetti ALG Moutinho ILD, da Silva EO, Kevin DJ. Cross-cultural Differences in Mental Health, Quality of Life, Empathy, and Burnout between US and Brazilian Medical Students. Academic Psychiatry. 2017. No prelo.

28. Tomaschewski-Barlem JG, Lunardi VL, Ramos AM, Silveira RS, Barlem ELD, Ernandes CM. Manifestações da síndrome de burnout entre estudantes de graduação em enfermagem. Enfermagem. 2013; 22 (3): 754-762.

29. Corral-Mulato S, Bueno SMV. (Des) conhecimento da Síndrome de Burnout entre acadêmicos de enfermagem. Revista de Enfermagem UERJ. 2014; 22 (2): 206-211.

30. Rueda FJM, Alves SMM, Baptista MN. Evidência de validade preliminar da escala de depressão (EDEP): um estudo com alunos de enfermagem. Psicologia Argumento. 2014; 32 (79): 107-117.

31. Tomaschewski-Barlem JG, Lunardi VL, Lunardi GL, Barlem ELD, Silveira RS, Vidal DAS. Burnout syndrome among undergraduate nursing students at a public university. Revista Latinoamericana de Enfermagem. 2014; 22 (6): 934-941.

32. da Silva RM, Goulart CT, Lopes LFD, Serrano PM, Costa ALS, de Azevedo Guido L. Hardy personality and burnout syndrome among nursing students in three Brazilian universities-an analytic study. BMC Nursing. 2014; 13 (1): 9-13.

33. Garbin ASC, Saliba AN, Santos RR, Prado RL, Garbin AJI. Burnout en estudiantes de odontología: evaluación a través mbi - versión estudiantes. Medicina y Seguridad del Trabajo. 2012; 58 (229): 327-334.

34. Nani BD, Lima PO, Marcondes FK, Groppo FC, Rolim GS, Moraes AB, Cogo-Muller K, Franz-Montan M. Changes in salivary microbiota increase volatile sulfur compounds production in healthy male subjects with academic-related chronic stress. PLoS ONE. 2017; 12 (3): e0173686.

\section{Como citar este artigo:}

Nassar LM, Andrade AMF, Arévalo JLS. Síndrome de burnout em estudantes de graduação dos cursos de medicina, enfermagem, odontologia e psicologia no brasil: uma revisão do panorama brasileiro. Rev. Aten. Saúde. 2018;16(57):98-109. 